\title{
A simplified and cost-effective enrichment protocol for the isolation of Campylobacter spp. from retail broiler meat without microaerobic incubation
}

Ping Zhou' ${ }^{1}$, Syeda K Hussain², Mark R Liles ${ }^{3}$, Covadonga R Arias ${ }^{4}$, Steffen Backert ${ }^{5}$, Jessica Kieninger ${ }^{1}$ and Omar A Oyarzabal ${ }^{1 *}$

\begin{abstract}
Background: To simplify the methodology for the isolation of Campylobacter spp. from retail broiler meat, we evaluated 108 samples (breasts and thighs) using an unpaired sample design. The enrichment broths were incubated under aerobic conditions (subsamples A) and for comparison under microaerobic conditions (subsamples M) as recommended by current reference protocols. Sensors were used to measure the dissolved oxygen (DO) in the broth and the percentage of oxygen $\left(\mathrm{O}_{2}\right)$ in the head space of the bags used for enrichment. Campylobacter isolates were identified with multiplex PCR assays and typed using pulsed-field gel electrophoresis (PFGE). Ribosomal intergenic spacer analyses (RISA) and denaturing gradient gel electrophoresis (DGGE) were used to study the bacterial communities of subsamples $M$ and $A$ after $48 \mathrm{~h}$ enrichment.
\end{abstract}

Results: The number of Campylobacter positive subsamples were similar for $\mathrm{A}$ and $\mathrm{M}$ when all samples were combined $(P=0.81)$ and when samples were analyzed by product (breast: $P=0.75$; thigh: $P=1.00)$. Oxygen sensors showed that DO values in the broth were around 6 ppm and $\mathrm{O}_{2}$ values in the head space were 14-16\% throughout incubation. PFGE demonstrated high genomic similarity of isolates in the majority of the samples in which isolates were obtained from subsamples A and M. RISA and DGGE results showed a large variability in the bacterial populations that could be attributed to sample-to-sample variations and not enrichment conditions (aerobic or microaerobic). These data also suggested that current sampling protocols are not optimized to determine the true number of Campylobacter positive samples in retail boiler meat.

Conclusions: Decreased DO in enrichment broths is naturally achieved. This simplified, cost-effective enrichment protocol with aerobic incubation could be incorporated into reference methods for the isolation of Campylobacter spp. from retail broiler meat.

\section{Background}

Campylobacteriosis in the most common foodborne disease in European countries, with an overall incidence of 47.6 cases per 100,000 population [1]; in Canada, with 36.1 cases every 100,000 person-years [2]; and the third most important bacterial foodborne diseases in the US [3]. Campylobacter spp. are found still at high prevalence in retail broiler carcasses in the US [4;5], and the

\footnotetext{
* Correspondence: oaoyarzabal@gmail.com

'Department of Biological Sciences, 1627 Hall Street, Alabama State

University, Montgomery, AL, USA

Full list of author information is available at the end of the article
}

isolation of Campylobacter spp. from clinical and food samples has always been done using microaerobic conditions, generally $85 \% \mathrm{~N}_{2}, 10 \% \mathrm{CO}_{2}$ and $5 \% \mathrm{O}_{2}$, during the enrichment of the samples and during the incubation of plate media. Different methods have been developed to generate microaerobic atmospheres and for a small number of samples, sachets that generate $\mathrm{CO}_{2}$ are commonly used [6]. If a larger number of samples are processed weekly, the evacuation-replacement is a more economical alternative. In this system, the air in the jar is partially removed by a vacuum pump and then replaced with a microaerobic gas mix. For a large 
number of samples, or to create unique microaerobic gas mixes with increased $\mathrm{H}_{2}$ content, more sophisticated microaerobic workstations have been developed [7].

Besides generating microaerobic conditions, several $\mathrm{O}_{2}$-quenching agents have been traditionally added to enrichment broths and agar plates for the isolation of Campylobacter spp. These agents neutralize the toxic effects of oxygen radicals and include blood or alkaline hematin [8;9], charcoal [10], iron salts and norepinephrine [11], and ferrous sulfate, sodium metabisulfite and sodium pyruvate (known as FBP supplement) [12]. In general, if blood or charcoal is added to agar plates, no other $\mathrm{O}_{2}$ quenching compounds are added [9]. To ensure the microaerobic gas mix for the length of incubation (at least $48 \mathrm{~h}$ ) sealed jars are commonly used, although plastic bags utilized to freeze food products with a "ziplock" type closing to prevent air leaks have been successfully used with gas-generating sachets and manual evacuation-replacement systems [13; 14].

Although a microaerobic mix is indispensable to grow Campylobacter spp. on agar plates, we have long suspected that no extra addition of any microaerobic gas mix is needed to keep Campylobacter spp. alive or even grow them in enrichment broths. In the present study we evaluated 108 retail broiler meat samples and compared the efficacy of Bolton broth incubated under microaerobic conditions using an evacuation-replacement system (subsamples $M$ ) versus incubation under aerobic conditions (subsamples A) for the isolation of naturally occurring Campylobacter spp. Presumptive Campylobacter spp. collected on agar plates were confirmed and identified with multiplex polymerase chain reaction (mPCR) assays and their DNA relatedness was analyzed using pulsed-field gel electrophoresis (PFGE). In addition, enriched broth cultures were analyzed with ribosomal intergenic spacer analysis (RISA) and denaturing gradient gel electrophoresis (DGGE) to determine the variability in the total bacterial population profiles of enrichment broths from subsamples $\mathrm{M}$ and $\mathrm{A}$. Our results indicate that microaerobic conditions that allow Campylobacter spp. to grow are naturally created in enrichment broths without the addition of extra microaerobic gas mix, and therefore a simplified method has been developed to identify these bacteria in food samples.

\section{Results}

Similar number of Campylobacter positive subsamples

From 108 retail broiler meat samples analyzed for the presence of Campylobacter spp., 48 (42\%) were positive from the microaerobic subsamples (subsamples $\mathrm{M})$, and 46 (44\%) were positive from the aerobic subsamples (subsamples A). Combining the data from subsamples $\mathrm{M}$ and A resulted in a total of 56 (52\%) positive samples for Campylobacter spp. Statistical comparison by chi-square showed that the number of Campylobacter positives from subsamples $\mathrm{M}$ and $\mathrm{A}$ were similar $(P>0.05)$, even when analyzing the subsamples by product (breasts or thighs) (Table 1 ). The sensitivity, specificity and accuracy were high (0.78 or above), and the Kappa values were above 0.50 for all comparisons, with the observed agreement in the Kappa value (considered the best agreement) always above 0.7 [15]. These high values reflected the large number of samples that were either positive (38 samples) or negative (52 samples) in both subsamples $M$ and A, as calculated by 2-by-2 tables (data not shown). Receiver operating characteristic (ROC) curves also showed that the true positive fraction was high and within the $95 \%$ confidence interval calculated for this dataset (Figure 1).

\section{mPCR assays identified both $C$. jejuni and $C$. coli species}

Table 2 shows the number of isolates collected and identified from subsamples $\mathrm{M}$ and $\mathrm{A}$, and for each product type. A $100 \%$ agreement was found between the mPCR assay described in Materials and Methods and the mPCR extensively used in our laboratories [16; 17]. All Campylobacter isolates were confirmed as either $C$. jejuni or C. coli, with C. jejuni comprising $83 \%$ and $85 \%$ of the isolates for subsamples $A$ and $M$, respectively. In 32 samples, subsamples $\mathrm{M}$ and $\mathrm{A}$ had $C$. jejuni, while six samples yielded $C$. coli in both subsamples. In 18 samples, only one of the subsamples (either $\mathrm{M}$ or $\mathrm{A}$ ) was positive for Campylobacter.

Table 1 Number of subsamples $M$ and $A$ that were positive for Campylobacter spp.

\begin{tabular}{llll}
\hline & $\begin{array}{l}\text { Campylobacter } \\
\text { Positive (\%) }\end{array}$ & & \\
\cline { 2 - 4 } $\begin{array}{l}\text { Enrichment } \\
\text { Conditions }\end{array}$ & Breast & Thighs & Total \\
\hline Microaerobic & $20(38)$ & $28(45)$ & 48 \\
Aerobic & $18(34)$ & $28(45)$ & 46 \\
Statistics & & & $(43)$ \\
$\chi^{2}$ a & 0.10 & & \\
P value & 0.75 & 0.00 & 0.50 \\
Sensitivity & 0.81 & 1.00 & 0.81 \\
Specificity & 0.78 & 0.88 & 0.79 \\
Accuracy & 0.80 & 0.85 & 0.87 \\
Kappa value & 0.58 & 0.86 & 0.83 \\
\hline
\end{tabular}

${ }^{a}$ A chi-square values $\leq 3.84$ assumes the null hypothesis that means from the reference method (microaerobic conditions) are equivalent to means from the test method (aerobic conditions) and cannot be rejected at the $5 \%$ level of confidence $(P<0.05)$. 


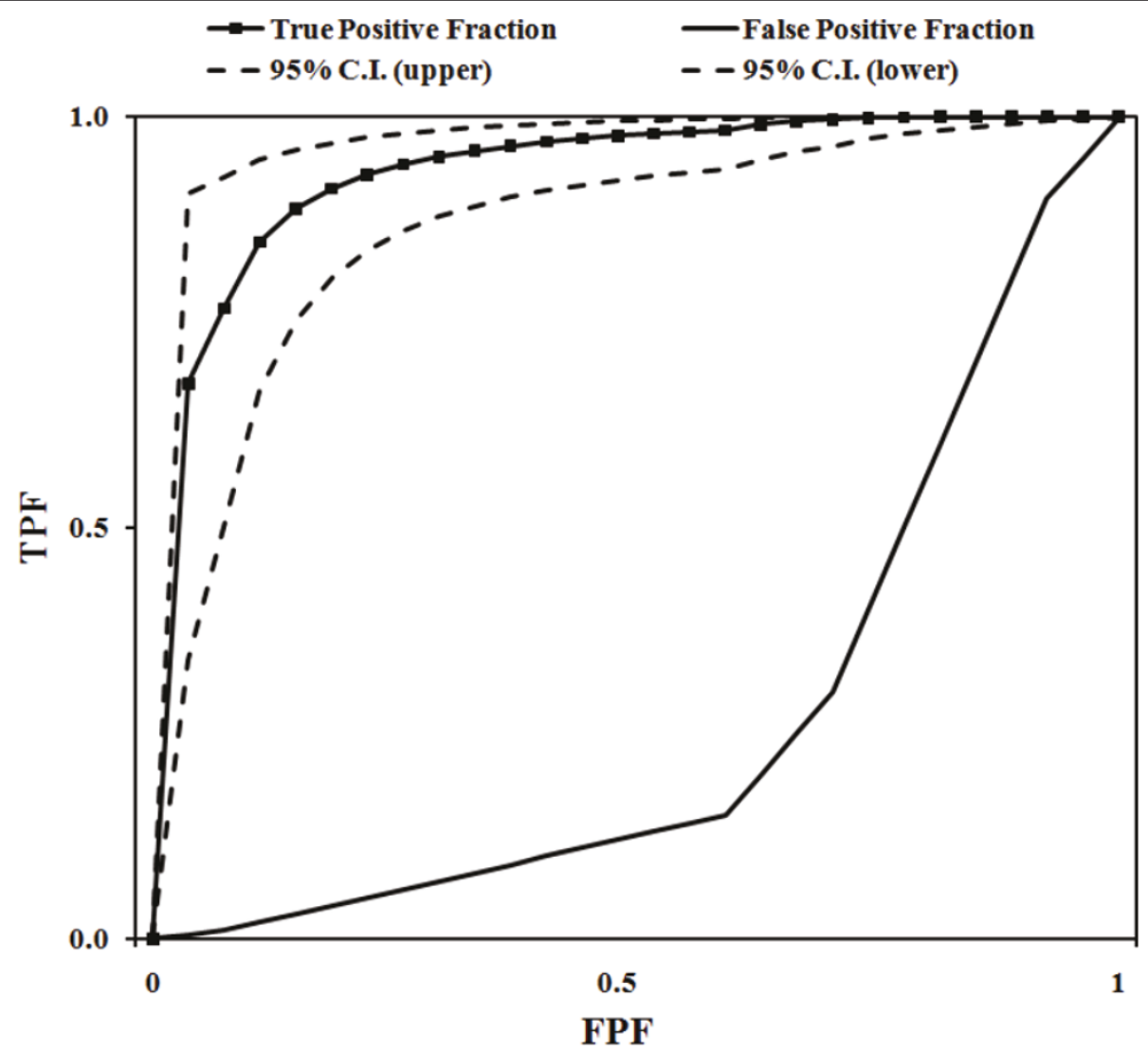

Figure 1 ROC curves. A high true positive fraction is shown with the upper and lower $95 \%$ confidence interval values. Consistent results were obtained from subsamples M (microaerobic conditions) and subsamples A (aerobic conditions) indicating that both methods were equivalent to isolate Campylobacter spp. from retail broiler meat.

PFGE similarity was high for most isolates collected from subsamples $\mathrm{M}$ and $\mathrm{A}$

PFGE analysis of 48 isolates (24 samples) showed a high genomic DNA relatedness between strains from subsamples $\mathrm{M}$ and the corresponding isolates from subsamples A (Figure 2). For 14 isolates (7 samples), the similarity between isolates from subsamples $\mathrm{M}$ and $\mathrm{A}$ was lower than $90 \%$ (Figure 3).

Table 2 Speciation of Campylobacter isolates using the mPCR assay described in Material and Methods and a previously described MPCR assay [17].

\begin{tabular}{lccccc}
\hline & & \multicolumn{2}{c}{$\begin{array}{c}\text { C. } \\
\text { jejuni }\end{array}$} & & \multicolumn{2}{c}{ C. coli } & \\
\cline { 3 - 6 } $\begin{array}{l}\text { Enrichment } \\
\text { Conditions }\end{array}$ & Total (\%) & Breast & Thighs & Breast & Thighs \\
\hline $\begin{array}{l}\text { Microaerobic } \\
\text { (subsamples M) }\end{array}$ & $48(44)$ & 19 & 22 & 1 & 6 \\
$\begin{array}{l}\text { Aerobic } \\
\text { (subsamples A) }\end{array}$ & $46(43)$ & 16 & 22 & 2 & 6 \\
\hline
\end{tabular}

Bacterial diversity measured by RISA and DGGE studies vary considerably among samples and subsamples

The results from the ARISA analysis of 41 subsamples $\mathrm{M}$ and 41 complimentary subsamples $\mathrm{A}$, chosen at random, showed a large variation in the microbial community and a lack of similarity patters intra- or intersample (Figure 4). Similar results were found using BioNumerics and the Pearson correlation to compare the band patterns of subsamples $M$ and A by DGGE. Even when analyzing the data using the Dice coefficient, which takes into account band migration, the results from subsamples $\mathrm{M}$ and A showed low DNA similarity at a cutoff point of $90 \%$ (data not shown). Table 3 shows the nearest neighbor identified from a BLASTn comparison of DGGE band sequences from subsamples $M$ and $A$. Sequencing information suggested that the bacteria present in most subsamples were facultative anaerobes and microaerobic organisms. BLAST results indicated a high degree of similarity of some rDNA amplicons (> 90\%) with Acinetobacter sp., Campylobacter jejuni, Lactobacillus sp. and Pseudomonas sp., and lower identity (80-90\%) with Lactobacillus sp. and 


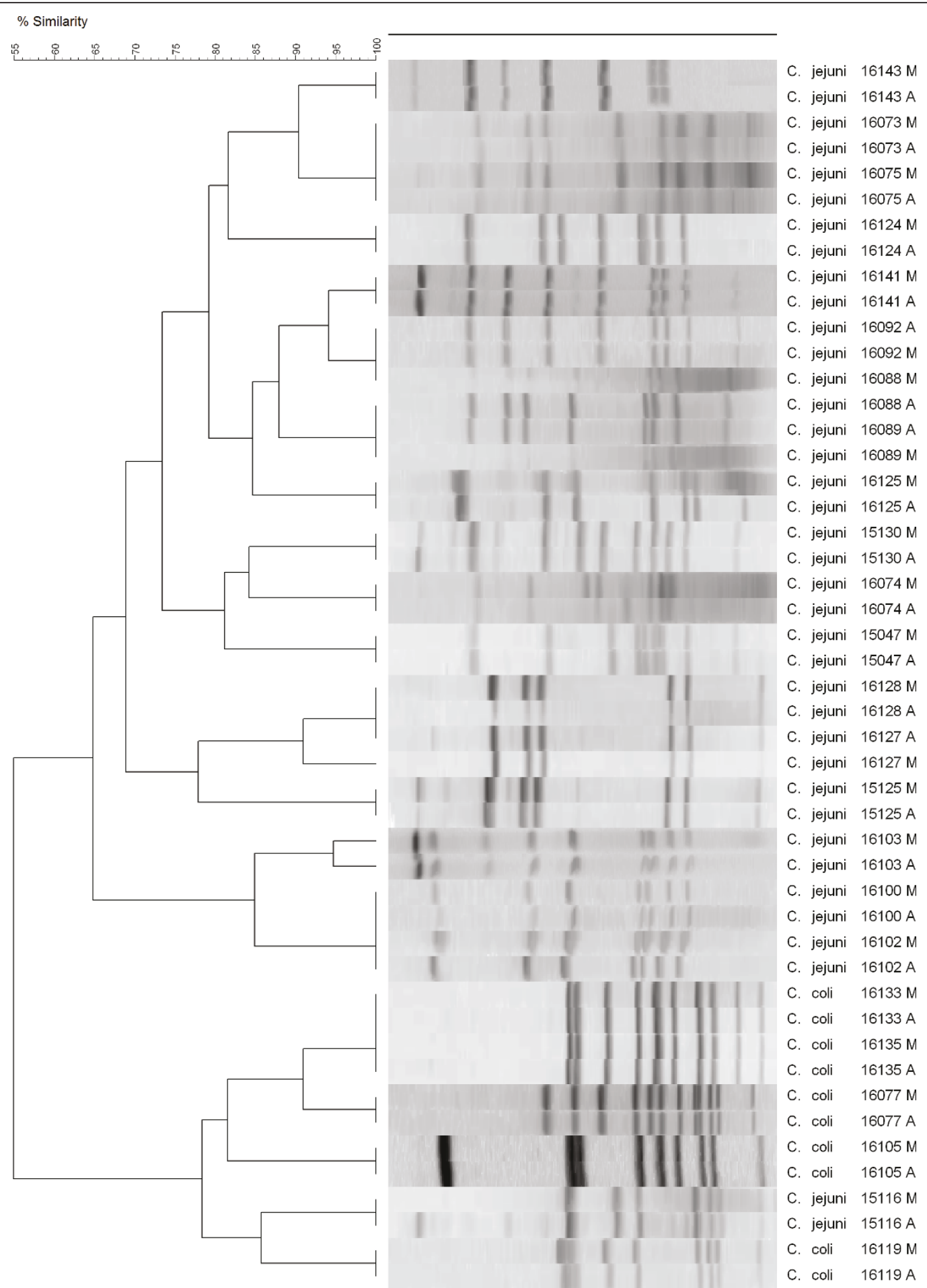

Figure 2 PFGE results. Isolates collected from subsamples M showing a high degree of similarity (>90\%) to isolates collected from subsample A. Pairwise comparisons were done using the Dice correlation and clustering analyses with the unweighted pair group mathematical average (UPGMA) clustering algorithm of BioNumerics ver. 5 (Applied Maths, Austin, TX, USA). The optimization tolerance was set at 2\% and the position tolerance for band analysis was set at $4 \%$. 


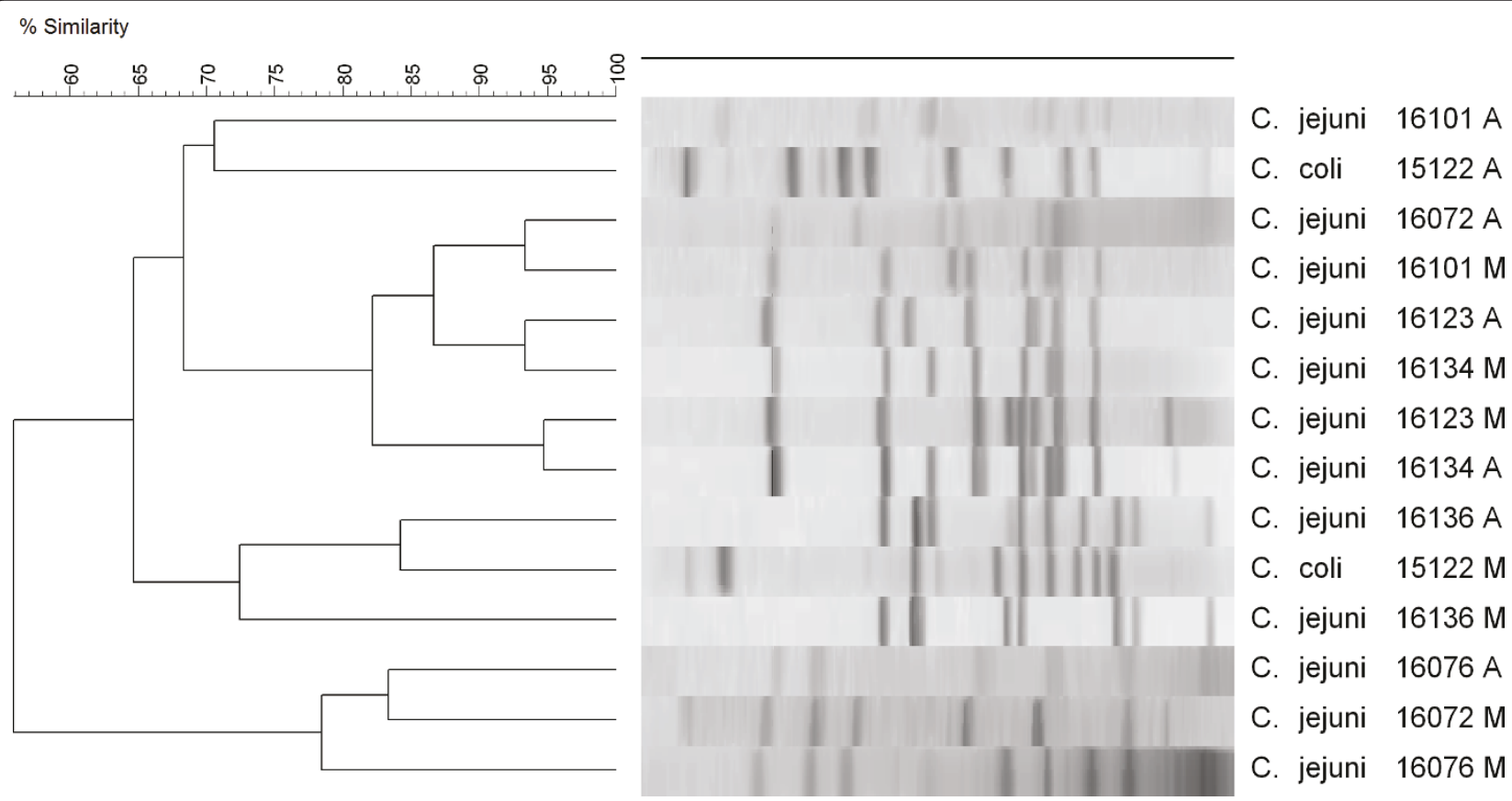

Figure 3 PFGE results. Isolates collected from subsamples M showing a low degree of similarity $(<90 \%)$ to isolates collected from subsample A. Pairwise comparisons and cluster analyses were done as described in Figure 2.

uncultured bacterial species. The sample that showed the largest bands separation had five well-distributed DNA bands in the gel (Table 3 A through E; DGGE bands K1 through K5). This sample was used consistently in DGGE gels as marker to normalize the gels and to allow for gel-to-gel comparisons using BioNumerics. A BLAST comparison showed that the sequences from these bands were similar to Acinetobacter sp. and Lactobacillus sp. (Table 3).

\section{$\mathrm{O}_{2}$ content decreased during the incubation of enrichment broths}

In samples incubated in Bolton broth without the addition of any microaerobic gas mix, the amount of $\mathrm{O}_{2}$ in the head space of the bags decreased over time and was at or below $17 \%$ at $24 \mathrm{~h}$ of incubation. The amount of $\mathrm{O}_{2}$ in the atmosphere was stable between 14 and $16 \%$ by $30 \mathrm{~h}$ of incubation; however, the amount of $\mathrm{O}_{2}$ never reached less than $14 \%$ (Figure 5). The amount of dissolved $\mathrm{O}_{2}$ in the enrichment broth, measured one inch from the bottom of the enrichment bags, reached 6 $\mathrm{ppm}$ at around $6 \mathrm{~h}$ of incubation. This value was stable thereafter and never reached above $7.5 \mathrm{ppm}$ (Figure 6). The presence of naturally occurring Campylobacter spp., either C. jejuni or C. coli, did not alter any of the values obtained with the sensors. In addition, incubation of 100 $\mathrm{ml}$ of Bolton broth without meat samples and without the addition of blood resulted in a similar pattern of DO values. In samples in which the $\mathrm{O}_{2}$ sensors were double bagged and gassed with a microaerobic gas mix, the DO decreased to around $5 \mathrm{ppm}$ and remained stable for up to $72 \mathrm{~h}$ (data not shown). Identical patterns of dissolved $\mathrm{O}_{2}$ levels were found when using ziplock plastic bags commonly used to freeze food products (The Glad Products Company, Oakland, CA) (data not shown).

\section{Discussion}

Several methods have been developed to generate microaerobic conditions for the growth and multiplication of Campylobacter spp. These methods are routine and are consistently used during the enrichment of food samples or during the incubation of inoculated plate media. However, little is known about the actual changes in $\mathrm{O}_{2}$ content in enrichment broth media during incubation $\left(37^{\circ} \mathrm{C}\right.$ or $\left.42^{\circ} \mathrm{C}\right)$. Our experiments were aimed at determining the changes of $\mathrm{O}_{2}$ content in the broth and in the air of the head space of the bags used to enrich the samples for the isolation of Campylobacter from retail broiler meat. The premises of this work was that the incubation of enrichment broth may naturally create microaerobiosis conducive to the grow of Campylobacter spp. Samples were therefore divided in two subsamples which were in turn incubated under microaerobic conditions $(\mathrm{M})$ or aerobic conditions (A).

We used an unpaired sample design, where the enrichment conditions differ between the reference (subsamples M) and the alternative method (subsamples 


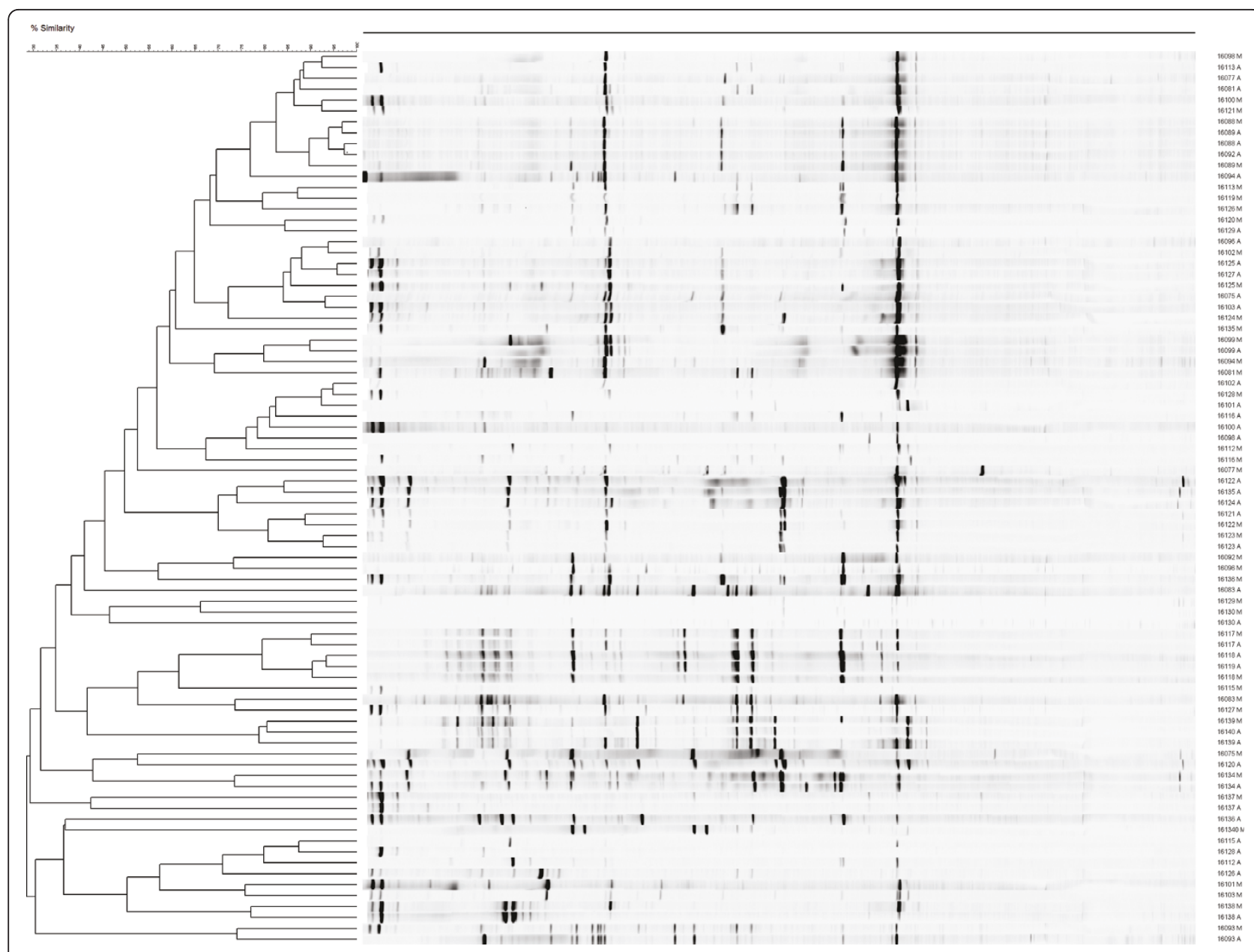

Figure 4 Results from RISA analysis. A low percentage of DNA similarity was found between the DNA profiles from subsamples M and the DNA profiles from subsamples $A$.

Table 3 Results from BLAST analysis of sequenced DGGE bands.

\begin{tabular}{llll}
\hline Marker & Band ID & $\begin{array}{l}\text { BLAST nearest homology } \\
\text { (GenBank accession number) }\end{array}$ & \% Identity \\
\hline A & K 1 & Acinetobacter sp. (FN563421) & 96 \\
B & K2 & Uncultured Myxococcales bacterium (FJ435015) & 93 \\
C & K 3 & Lactobacillus sp. L21 (AF159000) & 87 \\
D & K 4 & Lactobacillus sp. (FJ971864) & 95 \\
E & K 5 & Lactobacillus sp. JN4 (AF157041) & 90 \\
Microaerobic & subsample & Campylobacter jejuni (GQ479820) & 98 \\
& & Lactobacillus sp. 30A (FJ971864) & 98 \\
& & Pseudomonas sp. CB10 (EU482914) & 98 \\
Aerobic & Pseudomonas sp. R-35702 (AM886093) & 97 \\
& subsample & Campylobacter jejuni (GQ479820) & 98 \\
& & Lactobacillus sp. JN4 (AF157041) & 83 \\
& & Pseudomonas sp. CB11 (EU482915) & 98
\end{tabular}

Marker bands were used in all the gels.

${ }^{a}$ Unique DGGE bands from each subsample. 


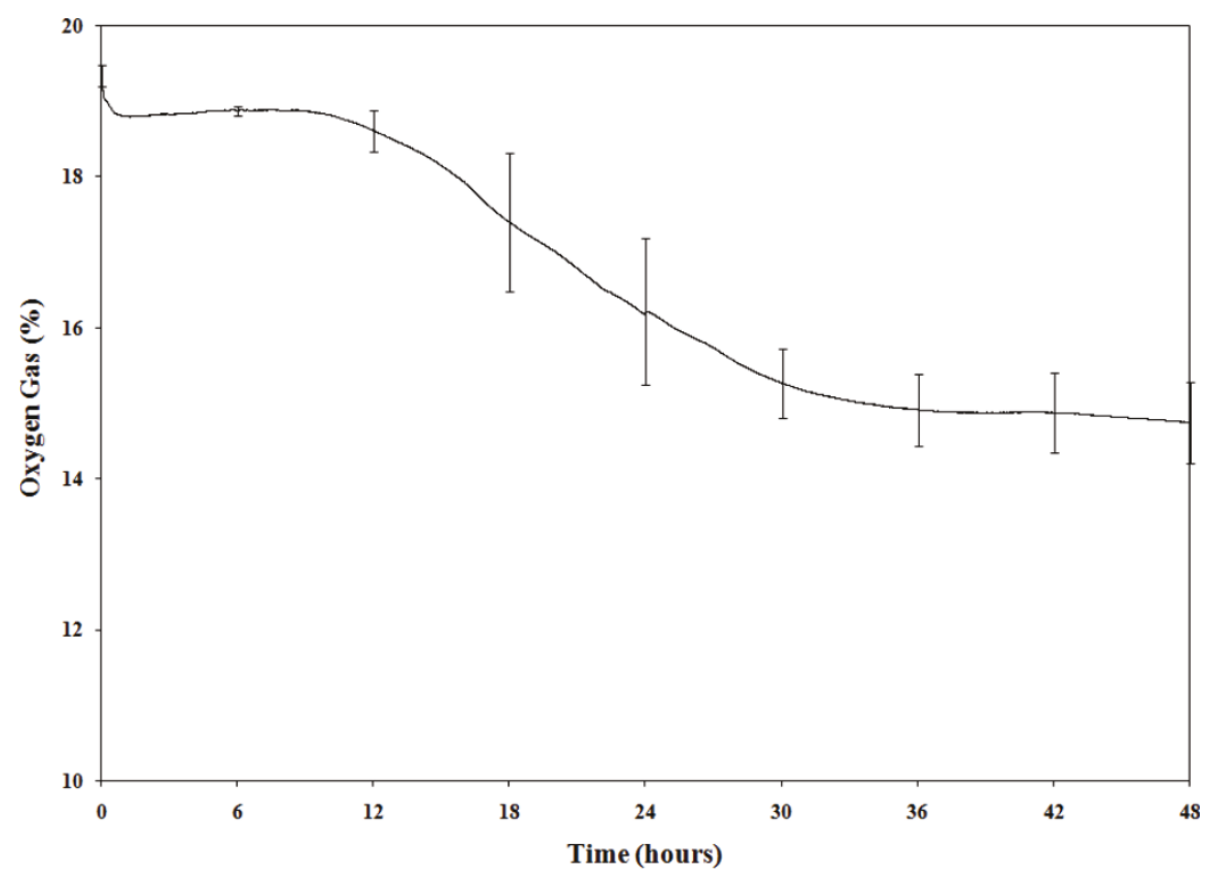

Figure 5 Oxygen measurements. Percentage of $\mathrm{O}_{2}$ in the head space of plastic bags throughout $48 \mathrm{~h}$ of incubation at $42^{\circ} \mathrm{C}$. Average $\pm \mathrm{SEM}$ of six measurements from subsamples positive for Campylobacter spp. after incubation under aerobic conditions. Measures were taken with an $\mathrm{O}_{2}$ sensor (Vernier, Beaverton, OR) as the percentage of $\mathrm{O}_{2}$ in the air in the head space.

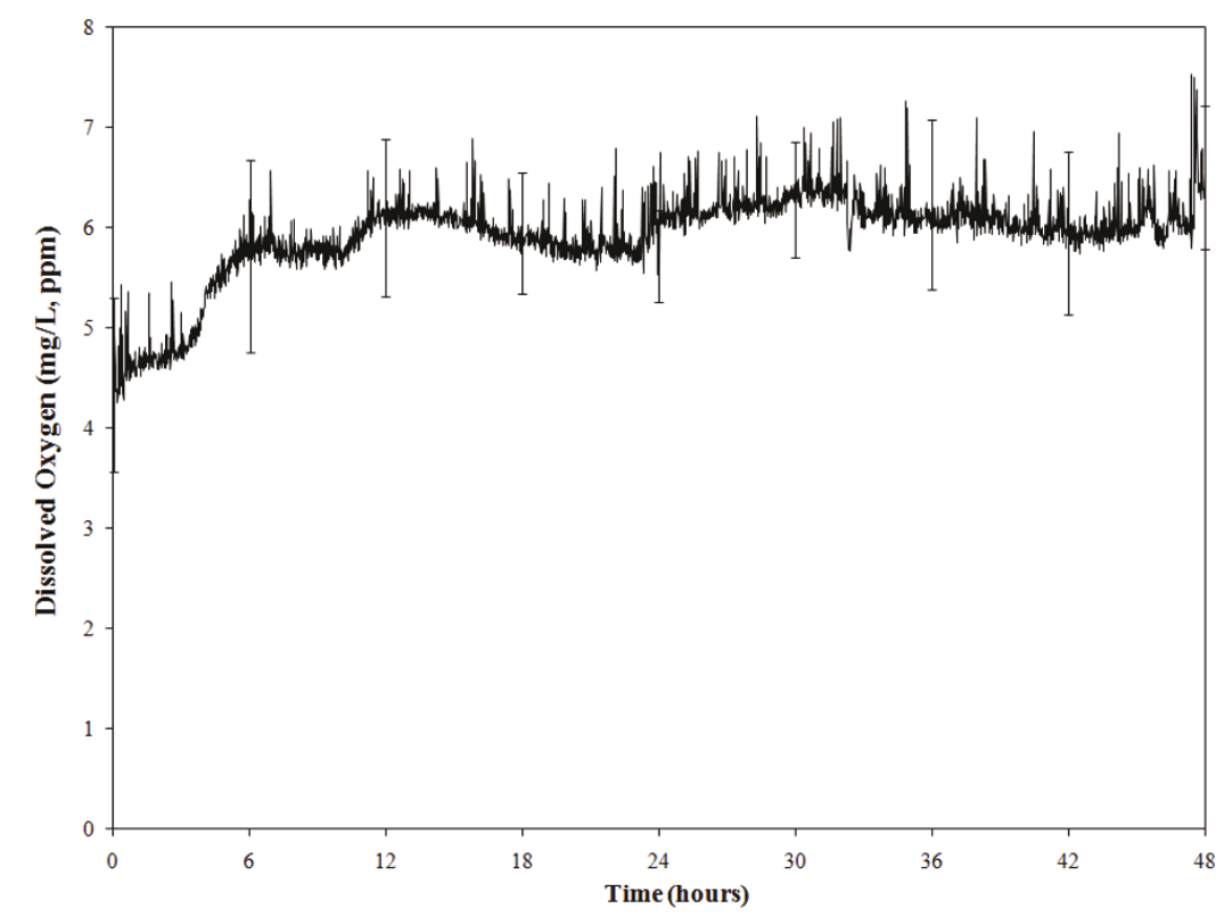

Figure 6 Oxygen measurements. Amount of dissolved oxygen (DO) in ppm in the enrichment broth. DO was measured at 1 inch from bottom of the bags, throughout $48 \mathrm{~h}$ of incubation at $42^{\circ} \mathrm{C}$. Average \pm SEM of six measurements from subsamples positive for Campylobacter spp. after incubation under aerobic conditions. Measurements were taken with a dissolved oxygen sensor (Vernier) and amount of oxygen in the liquid was recorded as $\mathrm{mg} / \mathrm{l}$ or ppm. 
A), and confirmed all presumptive positives using the same molecular protocols. Because the comparison of two qualitative methods is best accomplished near the limit of detection of these methods, we used naturally contaminated broiler meat samples, which have the lowest contamination that can be naturally found $[4 ; 17]$. The statistical analyses of data from unpaired samples are performed in the same way as for paired samples, mainly using McNemar's chi square test [18]. The number of Campylobacter positive subsamples was statistically similar between subsamples $\mathrm{M}$ and $\mathrm{A}$, and all isolates were clearly identified as $C$. jejuni or C. coli. These results demonstrate that enrichment broths incubated under normal, aerobic conditions are sufficient to detect Campylobacter spp. in retail broiler meat. There was an increase in number of total positive samples by $10 \%$ when combining the result of the two subsamples. These findings have been already reported several times for commercial broiler meat naturally contaminated with Campylobacter spp. [4; 17]. In addition, a ROC curve of the data showed a high true positive fraction, or rate, and a very low false positive fraction, which indicated a very strong correspondence in the results between the reference (subsamples $M$ ) and the alternative methods (subsamples A).

The traditional methodology of enriching $25 \mathrm{~g}$ of meat is the one suggested by the Bacteriological Analytical Manual of the Food and Drug Administration (FDA) [19], the Microbiology Laboratory Guidebook of the Food Safety and Inspection Services of the U. S. Department of Agriculture (FSIS UDSA) [20], the International Organization for Standardization [21], the Health Protection Agency of the UK [22], and several other countries' regulatory agencies. However, this methodology does not appear to be optimized to detect the true prevalence of Campylobacter spp. in retail broiler meat. PCR analysis of the isolates showed that $C$. jejuni or $C$. coli species are the only Campylobacter spp. found in retail broiler meat. Some samples can be contaminated with both species [17] but again the current methodology used in food samples is not accurate enough to reveal the extent of contamination of the same product with different Campylobacter strains. PFGE analysis further demonstrated that a single meat sample could be contaminated with two, or maybe more, isolates from the same species. For all practical purposes, C. jejuni and $C$. coli are the only two Campylobacter spp. found in retail poultry meat because no $C$. lari has been identified since the introduction of molecular techniques for routine identification of Campylobacter isolates, approximately 15 years ago [23]. The data collected with the $\mathrm{O}_{2}$ sensors showed that the amount of $\mathrm{O}_{2}$ in the enrichment broth was stable around 5-7 ppm after $6 \mathrm{~h}$ of enrichment. These $\mathrm{O}_{2}$ levels can be obtained by pressing out the air before closing the sample bags, and without the need of any vacuum, as is required when removing the air from a hard container. Whirl-Pak or ziplock bags performed similarly, showing that they are impervious to changes in the air trapped inside [13]. The fact that bags with only the enrichment broth (without meat or blood) created microaerobic conditions has encouraged us to continue this line of research, and we are currently testing other broths without blood to isolate Campylobacter spp. from retail broiler meat. Therefore, an inexpensive, simplified method can be developed for routinely use in the isolation and detection of Campylobacter spp. from food products.

Incubation of broth under normal aerobic conditions, with or without airspace, was done in the early 1980s to isolate Campylobacter spp. from fecal samples [24], and the use of $10 \% \mathrm{O}_{2}, 10 \% \mathrm{CO}_{2}$ and $80 \%$ of $\mathrm{N}_{2}$ facilitated and sustained the growth of Campylobacter spp. [25]. The ISO normative 10272-1:2006 requires a microaerobic environment but provides for an alternative incubation in a microaerobic atmosphere created by "screwcapped bottles or flasks filled with enrichment broth, leaving a headspace of less than $2 \mathrm{~cm}$, and tightly closing the caps" [21]. But much has been speculated about the need to have a higher surface area of the meat samples during enrichment to yield a higher number of positive samples under microaerobic conditions [26], or the exact depth of the airspace, the appropriate ratio of air to broth [27], and the correct type of incubation container to promote the growth of Campylobacter jejuni [28] to avoid significant difference in the results if a microaerobic atmosphere is not used [27]. Therefore, the microaerobic conditions are routinely used to isolate Campylobacter spp. However, our results do not suggest any correlation between surface and microaerobic conditions and do not support the notion that air to broth ratio and the type of container are indispensable to isolate Campylobacter spp. Our results point to the simple fact that any closed plastic bag naturally produces microaerobic environments conducive to the growth of Campylobacter spp. without the need to add any microaerobic gas mix. In our experiments, bags were closed to leave a minimum airspace and the samples were mixed, without stomaching, for few seconds. Thus, bags with subsamples $M$ had the same contact surface as bags with subsamples A.

The microbial population of the enriched samples in Bolton broth, as assessed by RISA and DGGE, was diverse. There are no current data on the microbial assemblage of retail broiler meat as a predictor to the presence of a bacterial pathogen, such as Campylobacter. Most of the work on the bacterial community of broiler meat was done more than 20 years ago using direct bacterial counts, and very few research studies 
have used culture-independent methods to study the microbial profile of these foods [29]. It is known, however, that some cold-tolerant bacteria, such as Enterobacteriaceae, Acinetobacter and Pseudomonas, are commonly present on broiler meat [30]. These bacteria are primarily facultative anaerobes or microaerobic organisms, and the ribosomal RNA gene sequences recovered in our samples, especially form the most prominent bands from DGGE gels, had a high similarity to these bacterial groups.

RISA and DGGE can be used to broadly characterize the total microbial population in complex samples. The results from these techniques were analyzed using the Pearson correlation, which is the standard procedure for comparison of densitometric curves $[31 ; 32]$. We analyzed the results with the Pearson correlation and also the Dice coefficient, which takes into account only the band position and not the band thickness, as it is the case in densitometric curves. Although the Dice correlation showed a higher DNA relatedness among corresponding $\mathrm{M}$ and $\mathrm{A}$ subsamples, the variability in the bacterial populations in each set of subsamples was still large and appeared to be more attributable to the original bacterial composition of the sampled meat itself than to the enrichment conditions (aerobic vs. microaerobic). A significant limitation of DGGE-derived phylogenetic data with the primers used in this study is the relatively short rDNA sequence obtained from each amplicon, thereby reducing the degree of phylogenetic inference that may be assigned to each band. Yet, both RISA and DGGE produced consistent results regarding the variability in the bacterial assemblages associated with retail broiler meat samples.

\section{Conclusions}

In summary, our results indicated that the enrichment of retail broiler meat at $42^{\circ} \mathrm{C}$ in closed plastic bags and without the addition of a microaerobic mix is adequate for the isolation of Campylobacter spp. With the advancement of DNA-based biosensors and automation for bacterial detection, enrichment broths could be screened for the presence of Campylobacter spp. in a shorter time, with greater sensitivity and without the generation of any microaerobic condition. In addition, food microbiology laboratories interested in establishing techniques for the isolation of Campylobacter from retail meat will have access to a cost-effective enrichment procedure without the need to invest in systems to generate microaerobiosis. Reference documents from the FDA and FSIS USDA should eventually be updated to provide for an alternative, simplified protocol that yields similar number of Campylobacter positive samples as the current reference protocols.

\section{Methods}

\section{Sample preparation, incubation and Campylobacter} isolation

Retail broiler meat samples (total $=108$ samples; 49 breasts and 59 thighs) were purchased from local stores (Auburn, AL) from April 2009 to October 2010. Samples were tested in batches of three to five samples per week. Each meat package was considered one sample, and from each package $\sim 1$-inch pieces were cut aseptically and mixed thoroughly. For all samples, $25 \mathrm{~g}$ of meat was weighed two times (two subsamples) in individual, sterile Whirl-Pak ${ }^{\circledR}$ (Nasco, Fort Atkinson, WI). Each subsample was enriched in $100 \mathrm{ml}$ of Bolton's broth (with antimicrobial supplements) and $5 \%(\mathrm{v} / \mathrm{v})$ of lysed horse blood [17]. The control subsamples (microaerobic subsamples) were incubated in anaerobic jars gassed with a microaerobic gas mix $\left(85 \% \mathrm{~N}_{2}, 10 \% \mathrm{CO}_{2}\right.$, $5 \% \mathrm{O}_{2}$; Airgas, Radnor, PA) using the evacuation-replacement system MACSmics Jar Gassing System (Microbiology International, Frederick, MD). The other subsamples (aerobic subsamples) were incubated without the addition of microaerobic gas mix, by closing the bags after removing the remaining air manually. All subsamples were incubated at $42^{\circ} \mathrm{C}$ for $48 \mathrm{~h}$.

After incubation and for all subsamples, $0.1 \mathrm{ml}$ of the enriched broth was transferred to modified charcoal cefoperazone deoxycholate agar [10] through a $0.65 \mu \mathrm{m}$ membrane filter as described elsewhere [33]. All agar plates were incubated under microaerobic conditions at $42^{\circ} \mathrm{C}$ for $48 \mathrm{~h}$. Presumptive Campylobacter colonies were observed under phase contrast microscopy (Olympus BX51, Olympus America Inc., Center Valley, P) for spiral morphology and darting motility. Presumptive isolates were stored at $-80^{\circ} \mathrm{C}$ in tryptic soy broth (Difco, Detroit, MI) supplemented with $20 \%$ glycerol (v/v) and $5 \%(\mathrm{v} / \mathrm{v})$ lysed horse blood for further analysis.

\section{Identification of presumptive Campylobacter isolates by MPCR assays}

Campylobacter isolates were recovered from frozen stocks by transferring to Brucella agar plates supplemented with $5 \%$ horse blood and through $0.6 \mu \mathrm{m}$ membrane filters as described above. Plates were incubated at $42^{\circ} \mathrm{C}$ under microaerobic conditions for $24 \mathrm{~h}$. Bacterial DNA was extracted using the Wizard ${ }^{\circledR}$ Genomic DNA Purification Kit as described by the manufacturer (Promega, Madison, WI), but bypassing the RNA digestion step. Isolates were identified with a previously described mPCR assay [17; 34; 35 , and a newly developed MPCR comprised of two sets of primers, one targeting the glyA gene of $C$. jejuni and the other targeting the ask gene of $C$. coli. Gene sequences downloaded from NCBI GenBank were aligned and analyzed using Molecular Evolutionary Genetics Analysis 
(MEGA) software [36] and primers were designed with the Integrated DNA Technologies PrimerQuest software. (Integrated DNA Technologies http://www.idtdna.com) The sequences of the primers are shown in Table 4. C. jejuni ATCC (American Type Culture Collection) 700819 and $C$. coli ATCC 43473 were used as control strains to set up the PCR conditions. The annealing temperatures of these primers were optimized with a gradient PCR program of a DNA ENgine $\left.{ }^{(}\right)$Thermal Cycler (Bio Rad laboratories, Hercules, CA), and the final conditions for this mPCR assay were 20 cycles of $94^{\circ} \mathrm{C}$ for 30 seconds; $63^{\circ} \mathrm{C}$ for 1 minute and $72^{\circ} \mathrm{C}$ for 1 minute. Amplified products were detected by standard gel electrophoresis in $1.5 \%$ agarose (Ultra Pure DNA Grade Agarose, Bio-Rad Laboratories) in tris-borate-EDTA buffer at $100 \mathrm{~V}$ for 40 minutes. DNA bands in the gels were stained with ethidium bromide and visualized using a VersaDoc ${ }^{\mathrm{TM}}$ Imaging System (Bio-Rad Laboratories).

\section{Typing of Campylobacter isolates with PFGE}

Isolates from 31 samples for which both subsamples were positive were randomly selected for PFGE analysis. Campylobacter isolates were typed using pulsed-filed gel electrophoresis (PFGE) following previously described protocols [16; 23]. Briefly, DNA was digested with SmaI and separated using a CHEF DR II system (Bio-Rad Laboratories, Hercules, CA) on 1\% agarose gels (SeaKem Gold agarose; Lonza). The DNA size marker used in the gels was Salmonella enterica subsp. enterica serovar Braenderup strain H9812 (ATCC BAA-664) restricted with $X b a I$. Restriction enzymes were purchased from New England BioLabs (Ipswich, MA). Gels were stained and visualized as described above (mPCR assays) and TIFF images were loaded into BioNumerics version 6 (Applied Maths, Austin, TX) for analysis. Pairwise-comparisons were done with the Dice correlation coefficient, and cluster analyses were performed with the unweighted pair group mathematical average (UPGMA) clustering algorithm. The optimization and position tolerance for band analysis were set at 2 and 4\%, respectively, and similarity among PFGE restriction patters was set at $90 \%$.

\section{DNA extraction from enrichment broths for bacterial population analysis}

DNA from enrichment broths after $48 \mathrm{~h}$ of incubation (subsamples $\mathrm{M}$ and $\mathrm{A}$ ) was extracted using the Wizard ${ }^{\circledR}$
Genomic DNA Purification Kit (Promega). To determine the microbial community profile of these subsamples, ribosomal intergenic spacer analysis (RISA) and denaturing gradient gel electrophoresis were performed (DGGE).

Forty-one sample sets chosen at random (22 negative for Campylobacter spp. in both subsamples and 19 positive for Campylobacter spp. in both samples [16 C. jejuni/C. jejuni and 3 C. coli/C. coli]) were analyzed by ARISA. RISA was generated by amplification of the internal spacer region (ISR) using the universal primers according to Cardinale et al. [37]. Amplified products were separated by electrophoresis on the NEN Global Edition IR2 DNA Analyzer (LI-COR, Lincoln, NE) following manufacturer's instructions. RISA images were processed with BioNumerics (Applied Maths). Following conversion, normalization, and background subtraction with mathematical algorithms, levels of similarity between fingerprints were calculated with the Pearson product-moment correlation coefficient $(r)$. Cluster analysis was performed using the UPGMA algorithm.

DGGE was performed using universal primers 338F (containing a 5' G+C clamp) and 518R, which amplify a segment of the 16S rDNA gene [38; 39]. PCR amplification consisted of 30 cycles of 5 min of denaturation at $94^{\circ} \mathrm{C}, 1 \mathrm{~min}$ of annealing at $55^{\circ} \mathrm{C}$, and $1 \mathrm{~min}$ of extension at $72^{\circ} \mathrm{C}$. The DGGE system (Ingeny phorU, Netherlands) had a denaturing gradient comprised of urea and formamide ranging from $45 \%$ to $65 \%$ in vertical polyacrylamide gels. Gels were stained with ethidium bromide and visualized under a UV gel imager. As a standard marker for gel comparison, every DGGE gel had one lane containing a DNA marker that had five specific bands. DGGE banding patterns were analyzed using BioNumerics (Applied Maths). Pairwise comparisons and cluster analysis were performed with the Pearson correlation coefficient and the Dice coefficient, and the UPGMA algorithm, respectively. The band position tolerance was set at $3 \%$ and a cut off value of $90 \%$ was used to determine similarity between subsamples. Selected bands from DGGE gels were excised and amplified using primers $338 \mathrm{~F}$ (without the $\mathrm{G}+\mathrm{C}$ clamp) and 518R. Amplicons were purified using the Wizard ${ }^{\circledR}$ SV Gel and PCR Clean-up System (Promega), and PCR products were sequenced with an ABI 3730 sequencer (Applied Biosystems, Foster City, CA) at Lucigen

Table 4 Primers developed in this study for the specific identification of $C_{\text {.jejuni }}$ and $C$. coli.

\begin{tabular}{|c|c|c|c|c|c|}
\hline Target Gene & Primer Name & Sequence $\left(5^{\prime}-3^{\prime}\right)$ & $\operatorname{Tm}\left({ }^{\circ} \mathrm{C}\right)$ & $\mathrm{G}+\mathrm{C}$ Content (\%) & Product Size (bp) \\
\hline \multirow[t]{2}{*}{ glyA } & F-JK & TGGCGGACATITAACTCATGGTGC & 59.6 & 50 & 264 \\
\hline & $\mathrm{R}-J \mathrm{~K}$ & CCTGCCACAACAAGACCTGCAATA & 59.5 & 50 & \\
\hline \multirow[t]{2}{*}{ ask } & F-JK & GGCTCCTITAATGGCCGCAAGATT & 59.8 & 50 & 306 \\
\hline & R-JK & AGACTATCGTCGCGTGATITAGCG & 58.5 & 50 & \\
\hline
\end{tabular}


Corporation (Middleton, WI). Sequences were aligned with MultAlin [40] and the consensus sequences were compared to the GenBank database using BLAST http:// blast.ncbi.nlm.nih.gov/Blast.cgi. The accession numbers of the sequences deposited in GenBank are GU250527 through GU250536.

\section{Detection of $\mathrm{O}_{2}$ changes during the incubation of enrichment broth}

The changes in the amount of $\mathrm{O}_{2}$ in the enrichment broth and the head space in the enrichment bags was measured in eight aerobic subsamples using a dissolved oxygen (DO) sensor (amount of oxygen in liquid measured as mg/l or ppm), and an oxygen $\left(\mathrm{O}_{2}\right)$ sensor (percentage of oxygen in the air). These sensors were purchased from Vernier (Beaverton, OR). A double bagging system was used to avoid air leaks during the measurements taken with the $\mathrm{O}_{2}$ sensors during incubation. Changes in $\mathrm{O}_{2}$ concentration were measured in all subsamples. The $\mathrm{O}_{2}$ Gas Sensor was calibrated to the environment within the plastic bag which produces condensation (100\% humidity), and therefore was started at $20.1 \mathrm{O}_{2}$ in percentage by volume. The DO sensor was positioned in the enrichment bag with the collection tip of the sensor placed at the bottom of the enrichment broth with the subsample. The $\mathrm{O}_{2}$ sensor was placed in the head space of the bag above the liquid. The excess air was expelled from the bag before sealing and incubation for $48 \mathrm{~h}$. The DO sensor was calibrated by prewarming the probe for $10 \mathrm{~min}$ in the broth before starting the readings. Throughout incubation, the sensors were connected to a laptop computer with the Logger Lite $^{\mathrm{TM}}$ data collection program (version 1.4 ) that recorded readings every $1 \mathrm{~min}$. The data were analyzed using Microsoft Excel (Microsoft Corporation, Redmond, WA).

\section{Statistical analyses}

An unpaired sample design was used where the number of Campylobacter positive subsamples enriched under microaerobic conditions (reference method) was compared to the number of Campylobacter positive subsamples enriched under aerobic conditions (alternative method). Statistical comparisons were made using the formula monemar. test $(x, y$, correct $=$ TRUE) of $\mathrm{R}$ [41], which is the McNemar's chi-squared $\left(\gamma^{2}\right)$ test for count data, and it is based on McNemar's Test for correlated proportions [42]. The accuracy, sensitivity, specificity, and Kappa values for the test were calculated using 2by-2 tables according to Hanrahan and Madupu [43]. A receiver operating characteristic (ROC) curve was determined with a web-based calculator with an ordinal rating scale of 1 through 4, where 1 represents samples that were negative for Campylobacter spp. in both subsamples, and 4 represents samples that were positive for both subsamples [44].

\section{Acknowledgements}

We thank Leslie Speegle for her assistance in collecting the sensor data and Kennedy Wekesa for allowing us access to the phase contrast microscope. JK work was supported by grant 0754966 from the Research Experiences for Undergraduates Program of the Biology Directorate of the National Science Foundation. The work of S.B. is supported by Science Foundation Ireland (UCD 09/IN.1/B2609).

\section{Author details}

${ }^{1}$ Department of Biological Sciences, 1627 Hall Street, Alabama State University, Montgomery, AL, USA. ²Department of Microbiology and Immunology, University of Arkansas for Medical Sciences, Little Rock, AK, USA. ${ }^{3}$ Department of Biological Sciences, 101 Rouse Life Science Bldg Auburn University, AL, USA. ${ }^{4}$ Department of Fisheries and Allied Aquacultures, 101 Swindle Hall, Auburn University, AL, USA. ${ }^{5}$ University College Dublin, UCD School of Biomolecular and Biomedical Sciences, Science Center West, Belfield Campus, Dublin 4, Ireland.

\section{Authors' contributions}

PZ carried out the sample collection, the DNA preparation, PFGE and PCRDGGE assays, and image statistical analysis. SKH helped with sample collection and DGGE analysis. ML helped optimize the DGGE analysis. CRA carried out RISA assays. SB helped analyze data and wrote part of the manuscript. JRK carried out the primer design to differentiate $C$. jejuni from C. coli. OAO conceived and coordinated the study, designed and revised the manuscript. All authors read and accepted the final version of the manuscript.

Received: 10 May 2011 Accepted: 3 August 2011

Published: 3 August 2011

\section{References}

1. Anon: European Food Safety Authority. Trends and sources of zoonoses, zoonotic agents and antimicrobial resistance in the European Union in 2004 2006, 96-16.

2. Anon: Isolation, identification, and enumeration of Campylobacter jejuni/ coli/lari from poultry rinse and sponge samples. Laboratory Guidebook, MLG 41.002010 [http://www.fsis.usda.gov/PDF/MLG_41_01.pdf].

3. Scallan E, Hoekstra RM, Angulo FJ, Tauxe RV, Widdowson MA, Roy SL, Jones JL, Griffin PM: Foodborne illness acquired in the United Statesmajor pathogens. Emerg Infect Dis 2011, 17:7-15.

4. Liu L, Hussain SK, Miller RS, Oyarzabal OA, Research Note: Efficacy of Mini VIDAS for the Detection of Campylobacter spp. from retail broiler meat enriched in Bolton broth with or without the supplementation of blood. J Food Prot 2009, 72:2428-2432.

5. Oyarzabal OA, Wesley IV, Barbaree JM, Lauerman LH, Conner DE: Specific detection of Campylobacter lari by PCR. J Microbiol Methods 1997, 29:97-102.

6. Reilly SS, Gilliland SE: Improving culturing techniques for Campylobacter. J Food Sci 2003, 68:2752-2757.

7. Frost JA, Oza AN, Thwaites RT, Rowe B: Serotyping scheme for Campylobacter jejuni and Campylobacter coli based on direct agglutination of heat-stable antigens. J Clin Microbiol 1998, 36:335-339.

8. Corry JE, Atabay HI, Forsythe SJ, Mansfield LR: Culture media for the isolation of campylobacters, helicobacters and arcobacters. In Handbook of culture media for food microbiology, progress in industrial microbiology. Edited by: Corry JE, Curtis G, Baird RM. Amsterdam, The Netherlands: Elsevier Science BV; 2003:271-316.

9. Corry JE, Post DE, Colin P, Laisney MJ: Culture media for the isolation of campylobacters. Int J Food Microbiol 1995, 26:43-76.

10. Hutchinson DN, Bolton FJ: Improved blood free selective medium for the isolation of Campylobacter jejuni from fecal specimens. J Clin Pathol 1984, 37:956-957.

11. Bowdre JH, Krieg NR, Hoffman PS, Smibert RM: Stimulatory effect of dihydroxyphenyl compounds on the aerotolerance of Spirillum volutans 
and Campylobacter fetus subspecies jejuni. Appl Environ Microbiol 1976 31:127-133.

12. George HA, Hoffman PS, Smibert RM, Krieg NR: Improved media for growth and aerotolerance of Campylobacter fetus. J Clin Microbiol 1978, 8:36-41.

13. Oyarzabal OA, Macklin KS, Barbaree JM, Miller RS: Evaluation of agar plates for direct enumeration of Campylobacter spp. from poultry carcass rinses. Appl Environ Microbiol 2005, 71:3351-3354.

14. Szalanski AL, Owens CB, Mckay T, Steelman CD: Detection of Campylobacter and Escherichia coli 0157:H7 from filth flies by polymerase chain reaction. Med Vet Entomol 2004, 18:241-246.

15. Fleiss $J$, Levin B, Paik MC: The measurement of interrater agreement. In Statistical methods for rates and proportion.. 3 edition. Edited by: Fleiss JL, Levin B, Paik MC. New York, NY: Wiley-Interscience; 2003:603-617.

16. Behringer M, Miller WG, Oyarzabal OA: Typing of Campylobacter jejuni and Campylobacter coli isolated from live broilers and retail broiler meat by flaA-RFLP, MLST, PFGE and REP-PCR. J Microbiol Methods 2010, 84:194-201.

17. Oyarzabal OA, Backert S, Nagaraj M, Miller RS, Hussain SK, Oyarzabal EA: Efficacy of supplemented buffered peptone water for the isolation of Campylobacter jejuni and C. coli from broiler retail products. J Microbiol Methods 2007, 69:129-136.

18. Brunelle S: Validation of microbiological methods for food. Statistical Aspects of the Microbiological Examination of Foods. 2 edition. Academic Press, Elsevier; 2008, 259-277.

19. Hunt JM, Abeyta C, Tran T: Isolation of Campylobacter Species from Food and Water. In Food and Drug Administration Bacteriological Analytical Manual. Volume Chapter 7.. 8 edition. Revision A/1998. Arlington. VA. Association of Official Analytical Chemists International; 2001.

20. Anon: Isolation, identification, and enumeration of Campylobacter jejuni/ coli/lari from poultry rinse and sponge samples. Laboratory Guidebook MLG 41.002010 [http://www.fsis.usda.gov/science/

Microbiological_Lab_Guidebook/].

21. Anon: Microbiology of food and animal feeding stuffs - Horizontal method for detection and enumeration of Campylobacter spp. Part 1 Detection methods. ISO 2006, 10272-1:2006.

22. Anon: Detection of Campylobacter species. National Standard Method F21 Health Protection Agency. UK; 1998, 2.

23. Miller RS, Miller WG, Behringer MG, Hariharan $H$, Matthew V, Oyarzabal OA: DNA identification and characterization of Campylobacter jejuni and Campylobacter coli isolated from caecal samples of chickens in Grenada. J Appl Microbiol 2010, 108:1041-1049.

24. Martin WH, Patton CM, Morris GK, Potter ME, Puhr ND: Selective enrichment broth medium for isolation of Campylobacter jejuni. J Clin Microbiol 1983, 17:853-855

25. Bolton FJ, Coates D: Development of a blood-free Campylobacter medium: screening tests on basal media and supplements, and the ability of selected supplements to facilitate aerotolerance. J App/ Microbiol 1983, 54:115-125.

26. Wesley RD, Swaminathan B, Stadelman WJ: Isolation and enumeration of Campylobacter jejuni from poultry products by a selective enrichment method. Appl Environ Microbiol 1983, 46:1097-1102.

27. Moran L, Kelly C, Madden RH: Factors affecting the recovery of Campylobacter spp. from retail packs of raw, fresh chicken using ISO 10272-1:2006. Lett Appl Microbiol 2009, 48:628-632.

28. Reilly SS, Gilliland SE: Improving culturing techniques for Campylobacter. Food Sci 2003, 68:2752-2757.

29. Vihavainen E, Lundstrom HS, Susiluoto T, Koort J, Paulin L, Auvinen P, Björkroth KJ: Role of broiler carcasses and processing plant air in contamination of modified-atmosphere packaged broiler products with psychrotrophic lactic acid bacteria. Appl Environ Microbiol 2007, 73:1136-1145.

30. Vihavainen EJ, Björkroth J: Microbial ecology and spoilage of poultry meat and poultry meat products. In Handbook of Poultry Science and Technology, Secondary Processing. Volume 2. Edited by: Guerrero-Legarreta et al. New York. NY: Blackwell-Wiley Publishing; 2010:485-493.

31. Vauterin L, Vauterin P: Integrated databasing and analysis. In Molecular identification, systematic and population structure of prokaryotes. Edited by: Stackebrandt E. Berlin Heidelberg Springer-Verlag; 2006:141-217.

32. Tzeneva VA, Heilig HG, Akkermans HJ, van Vliet W, Akkermans ADL, de Vos WM, Smidt H: 16S rRNA targeted DGGE fingerprinting of microbial communities. In Environmental Genomic. Edited by: Cristofre MC. Totowa, NJ, Humana Press; 2007:335-349.

33. Speegle L, Miller ME, Backert S, Oyarzabal OA, Research Note: Use of cellulose filters to isolate Campylobacter spp. from naturally contaminated retail broiler meat. J Food Prot 2009, 72:2592-2596.

34. Linton D, Lawson AJ, Owen RJ, Stanley J: PCR detection, identification to species level, and fingerprinting of Campylobacter jejuni and Campylobacter coli direct from diarrheic samples. J Clin Microbiol 1997, 35:2568-2572

35. Persson S, Olsen KEP: Multiplex PCR for identification of Campylobacter coli and Campylobacter jejuni from pure cultures and directly on stool samples. J Med Microbiol 2005, 54:1043-1047.

36. Anon: Molecular Evolutionary Genetics Analysis MEGA Version 4. 2010 [http://www.megasoftware.net]

37. Cardinale M, Brusetti L, Quatrini P, Borin S, Puglia AM, Rizzi A, Zanardini E, Sorlini C, Corselli C, Daffonchio D: Comparison of different primer sets for use in automated ribosomal intergenic spacer analysis of complex bacterial communities. Appl Environ Microbiol 2004, 70:6147-6156.

38. Muyzer G, De Waal EC, Uitterlinden AG: Profiling of complex microbial populations by denaturing gradient gel electrophoresis analysis of polymerase chain reaction-amplified genes coding for $16 \mathrm{~S}$ rRNA. Appl Environ Microbiol 1993, 59:695-700.

39. Sheffield VC, Cox DR, Lerman LS, Myers RM: Attachment of a 40-base-pair $\mathrm{G}+\mathrm{C}$-rich sequence GC-clamp to genomic DNA fragments by the polymerase chain reaction results in improved detection of single-base changes. Proc Natl Acad Sci USA 1989, 86:232-236.

40. Corpet F: Multiple sequence alignment with hierarchical clustering. Nucleic Acids Res 1988, 16:10881-10890.

41. Anon: R: A language and environment for statistical computing. $R$ Foundation for Statistical Computing, Vienna, ISBN 3-900051-07-0 R Development Core Team. Austria; 2010 [http://www.R-project.org]

42. McNemar Q: Note on the sampling error of the difference between correlated proportions or percentages. Psychometrika 1947, 12:153-157.

43. Hanrahan EJ, Madupu G: The 2-by-2 table and its concepts. In Appleton \& Lange's review of epidemiology \& biostatistics for the USMLE. Edited by: Hanrahan EJ, Madupu G. New Jersey: Prentice Hall. Englewood Cliffs; 1994:11-19.

44. Eng J: Web-based calculator for ROC curves. 2007, Accessed from http:// www.rad.jhmi.edu/jeng/javarad/roc/JROCFITi.html. ROC Analysis.

doi:10.1186/1471-2180-11-175

Cite this article as: Zhou et al:: A simplified and cost-effective enrichment protocol for the isolation of Campylobacter spp. from retail broiler meat without microaerobic incubation. BMC Microbiology 2011 $11: 175$.

\section{Submit your next manuscript to BioMed Central and take full advantage of:}

- Convenient online submission

- Thorough peer review

- No space constraints or color figure charges

- Immediate publication on acceptance

- Inclusion in PubMed, CAS, Scopus and Google Scholar

- Research which is freely available for redistribution

Submit your manuscript at www.biomedcentral.com/submit
C Biomed Central 